LIAMES, Campinas, SP, v. 20, 1-21, e020015, 2020

\title{
Elementos de la morfofonología del chiquitano migueleño ${ }^{1}$
}

\author{
Andrey Nikulin \\ Pesquisador colaborador, Universidade de Brasília, Brasil \\ https://orcid.org/0000-0003-2237-564X
}

\begin{abstract}
In this paper, I describe several morphophonological processes and a morphophonological constraint attested in the variety of Chiquitano (Macro-Jê) spoken in San Miguel de Velasco (Santa Cruz, Bolivia), based on original fieldwork data obtained from five native speakers. I present a detailed description of the longdistance nasalization of continuant consonants and high vowels; the affrication of $|\mathrm{t} k|$ in certain environments; the progressive palatalization of $|\mathrm{p} \beta \mathrm{m} \mathrm{t} \mathrm{kx}|$; the minimality constraints which apply to nominal and adpositional stems; five types of vowel harmony which affect specific affixes or clitics; the process of raising of $|\mathrm{o}|$ to $/ \mathrm{u} /$ preceding a syllable which contains an /a/; the process of lowering of $|i|$ to $/ \varepsilon /$ before $/ \mathrm{s}$ ts/; the morphophonological elision of high vowels. Some of these processes are shared by other varieties of Chiquitano, whereas others lack any known equivalent in dialects other than Migueleño.
\end{abstract}

KEYwORDS: Morphophonology; Chiquitano; Phonological processes; Palatalization; Macro-Jê.

RESUMEN: Este artículo describe algunos de los procesos morfofonológicos y una restricción morfofonológica atestiguados en la variedad del idioma chiquitano (macro-ye) hablada en San Miguel de Velasco (Santa Cruz, Bolivia), con base en datos recogidos en campo con cinco hablantes nativos. Se describen detalladamente la nasalización a distancia de las consonantes continuas y de las vocales altas; la africación de $\mid \mathrm{t}$ k| en determinados ambientes; la palatalización progresiva de $\left|\mathrm{p} \beta_{\mathrm{r}} \mathrm{m} \mathrm{t} \mathrm{k} \mathrm{x}\right|$; las restricciones de minimalidad que aplican a los temas nominales y adposicionales; cinco tipos de armonía vocálica que afectan afijos o clíticos específicos; el proceso de elevación de $|\mathrm{o}| \mathrm{a} / \mathrm{u} /$ delante de una sílaba que contiene /a/; el proceso de descenso de $|\mathrm{i}| \mathrm{a} / \mathrm{E} /$ delante de /s ts/; la elisión morfofonológica de vocales orales altas. Algunos de los procesos documentados son compartidos por otras variedades del chiquitano, mientras que otros no tienen equivalentes conocidos en los demás dialectos. Palabras Clave: Morfofonología; Chiquitano; Procesos fonológicos; Palatalización; Lenguas macro-ye.

\section{Introducción}

En este artículo describo los fenómenos morfofonológicos más importantes que ocurren en el chiquitano migueleño (Glottocode 4.3 [sanm1288]), una variedad lingüística

${ }^{1}$ Este estudio habría sido imposible sin la colaboración de las personas que me han enseñado el chiquitano migueleño: Doña Ignacia (Násiya) Yopié Tomichá, Doña Antonia (Ato 'ĩxh) Socoré Masaí, Don Victoriano Julián Laverán Ramos (in memoriam), Doña Micaela Ribera Montero y Don Miguel Putaré Tapanaché (in memoriam). Agradezco a dos evaluadores/as anónimos/as de LIAMES y a Andrés Pablo Salanova por sus comentarios y sugerencias a este manuscrito. Finalmente, doy mis gracias a Brittany Hause y Luca Ciucci, con quienes he discutido varios puntos problemáticos en el análisis. Evidentemente, soy el único responsable de eventuales inexactitudes o errores. 


\section{NIKULIN - ELEMENTOS DE LA MORFOFONOLOGÍA...}

hablada en el cantón de San Miguel de Velasco, en la segunda sección de la provincia J. M. de Velasco del departamento de Santa Cruz, Bolivia. ${ }^{2}$ El chiquitano migueleño está estrechamente relacionado a otras variedades utilizadas en el Oriente boliviano, como el bésiro (hablado en la región de Lomerío, en Concepción y en San Javier) y el chiquitano oriental (recordado por algunas personas en San Ignacio de Velasco, San Rafael de Velasco, Santa Ana y Santiago de Chiquitos, así como en algunas comunidades en la zona fronteriza del estado de Mato Grosso, Brasil). Juntas, componen la macrolengua chiquitana (ISO 639-3 [cax]), que, a su vez, es un probable miembro de la agrupación lingüística macro-ye (Adelaar 2008); alternativamente, se la ha clasificado como paramacro-ye (Nikulin 2020).

El chiquitano migueleño no ha sido previamente descrito o documentado, salvo en dos cortos artículos de este autor acerca de particularidades morfológicas de esa variedad (Nikulin 2019a, b) y un esbozo de su fonología segmental (Nikulin, por aparecer), en que además se discute en algún detalle su situación sociolingüística. El dialecto migueleño presenta una serie de diferencias sistemáticas de las demás variedades documentadas del chiquitano en todos los niveles lingüísticos. En la fonología, es el único en poseer en su inventario la fricativa velar / $\mathrm{x}$ / (correspondiéndole a la fricativa retrofleja /s/ en las demás variedades del chiquitano) y la oclusiva pospalatal / / (resultado de la palatalización de las oclusivas $|\mathrm{p}| \mathrm{y}|\mathrm{k}|$, que en los demás dialectos se palatalizan para $\left[\mathrm{p}^{\mathrm{j}}\right]$ y $\left[\mathrm{k}^{\mathrm{j}}\right]$ o bien no se palatalizan), como en la palabra $\left|\mathrm{i}_{1}-\mathrm{pa}-\mathrm{x} \dot{\mathrm{i}}\right| / \mathbf{i} \underline{\text { cáxít }} /$ [i'cax] 'su brazo, su ala' (= bésiro /(n)ipáşi/ [(n)i'pjas]). ${ }^{3}$ En la morfología, difiere de todos los demás dialectos documentados en poseer dos prefijos distintos de la primera persona del singular, la escogencia entre los cuales está condicionada por el generolecto (|ix-| en el habla femenina, |ij-| en el habla masculina), como en |ix-ikiki|/6ikíki/ [6i'kiki] 'mi uña.

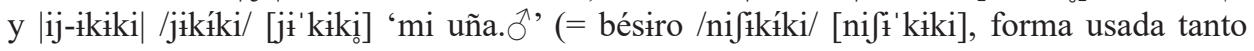
por los hombres como por las mujeres). Además, el migueleño parece ser la única variedad del chiquitano en que los sufijos del plural nominal y del sujeto locufórico (es decir, de primera o segunda persona) de un verbo finito no son homónimos: para el primer morfema se constata la forma /-kaa/ [-ka], mientras que para el segundo se ha atestiguado la forma /-ka/ [ka]; en todas las demás variedades del chiquitano se registran formas idénticas (/-ka/ [-ka]). Todas estas características conllevan la necesidad de estudiar el chiquitano migueleño separadamente de las demás variedades de la misma macrolengua.

El objetivo de este estudio es proporcionar una descripción de los procesos morfofonológicos que caracterizan el chiquitano migueleño, sin pretensión de completud. En particular, no discutiré la morfofonología del acento (Nikulin, entregado para

${ }^{2}$ Los datos utilizados en este artículo provienen del trabajo de campo realizado por el autor en cinco visitas (julio de 2017, enero de 2018, junio de 2018, febrero de 2019, julio de 2019) a las comunidades de San Juan de Lomerío y San Miguel de Velasco. El corpus incluye aproximadamente 40 horas de grabaciones tanto de elicitación como de habla natural; en total se trabajó con cinco hablantes.

${ }^{3}$ Utilizo las siguientes convenciones en este artículo. Las representaciones morfofonológicas (subyacentes) se citan entre plecas $(|\ldots|)$, las fonológicas entre barras oblicuas $(/ \ldots /)$ y las fonéticas entre corchetes ([...]). Para los archifonemas en el nivel morfofonológico, se usa la notación fraccionaria, con una posibilidad indicada en el numerador y otra en el denominador. Los símbolos fonéticos son los del Alfabeto Fonético Internacional, con la excepción de la tilde ('), que marca la posición del acento en las representaciones fonológicas. Las formas del bésiro se citan según Parapaino Castro (2008), a no ser que se indique lo contrario. 
publicación) ni la compleja interacción entre los prefijos de persona/número y los prefijos que se interponen entre estos últimos y la raíz. Considerando que el chiquitano migueleño aún no ha sido objeto de descripciones lingüísticas, se juzgó oportuno que este trabajo tuviera un carácter esencialmente descriptivo - y no teórico o comparativo-, para que los datos del chiquitano migueleño puedan ser más fácilmente aprovechados por otros autores en sus investigaciones tipológicas o teóricas, independientemente de la teoría fonológica que adopten. Por razones de espacio, se optó por no incluir una comparación sistemática de los procesos morfofonológicos del dialecto migueleño con los de las demás variedades del chiquitano, aunque en determinadas ocasiones se comentan las diferencias interdialectales más notables.

Al hablar del nivel fonológico o de fonemas en este artículo, me refiero a un nivel de representación relativamente superficial donde se representan los contrastes y a partir del cual se puede obtener toda la gama de realizaciones fonéticas mediante las reglas automáticas (tanto obligatorias como opcionales), analizadas detalladamente en Nikulin (por aparecer), que no interactúan de ningún modo con la estructura morfológica de la palabra y por ello son consideradas reglas posléxicas (cf. Kiparsky 1982). En cambio, el nivel morfofonológico al que me refiero en las representaciones subyacentes es el nivel en que cada morfema posee una forma fija; es el nivel más profundo que se puede proyectar de los datos sincrónicos. Considero que el inventario de segmentos en ese nivel puede ser distinto del inventario fonológico (es decir, no acepto la restricción a la preservación de estructura; cf. Harris 1987). En particular, considero que los segmentos $/ \underline{\underline{j}} \underline{\mathrm{c}} 6 \mathrm{w} \mathrm{y} /$, que integran el inventario fonológico del chiquitano migueleño, no son parte de su inventario morfofonológico; en un sentido inverso, represento como segmentos subyacentes distintos las unidades que normalmente corresponden a un solo fonema pero que presentan diferencias en su comportamiento morfofonológico $\left(\left|\mathrm{i}_{1}\right| \mathrm{e}\left|\mathrm{i}_{2}\right| ;|\beta| \mathrm{y} \mid\left(\beta_{\mathrm{r}} \mid\right)\right.$. Además, admito el uso de archifonemas en los casos en que el análisis es ambiguo como consecuencia de una neutralización.

La organización de este artículo es como sigue. El inventario fonológico del chiquitano migueleño se presenta en el apartado 1. Los apartados 2-4 abordan los procesos morfofonológicos que afectan los segmentos consonánticos: la nasalización, la africación y la palatalización. En el apartado 5 se discutirá una restricción que determina el tamaño mínimo de los temas nominales y adposicionales del chiquitano migueleño. Los procesos morfofonológicos que afectan las vocales (la armonía vocálica en afijos y clíticos específicos, la elevación de $|\mathrm{o}| \mathrm{a} / \mathrm{u} /$, el descenso de $|\mathrm{i}| \mathrm{a} / \varepsilon /$ y la elisión morfofonológica) se presentarán en los apartados 6-9. En el apartado 10 se ofrece un breve resumen de la discusión en las secciones anteriores.

\section{Inventario fonológico}

En este apartado, presento las informaciones básicas acerca de la fonología segmental del chiquitano migueleño. El inventario de los segmentos consonánticos contrastivos se reproduce en la Tabla 1. 
NIKULIN - ELEMENTOS DE LA MORFOFONOLOGÍA...

Tabla 1. Los fonemas consonánticos del chiquitano migueleño (Nikulin, por aparecer)

\begin{tabular}{|l|c|c|c|c|c|c|c|}
\hline & labiales & dentoalveolares & postalveolar & retrofleja & palatales & velares & glotales \\
\hline obstruyentes & $\mathrm{p}$ & $\mathrm{t}$ & $\underline{\mathrm{t}}$ & & $\underline{\mathrm{c}}$ & $\mathrm{k}$ & $?$ \\
\hline africadas & & $\mathrm{ts}$ & & & $\mathrm{t} \int$ & & \\
\hline fricativas & & $\mathrm{s}$ & & $\mathrm{s}$ & $\mathrm{c}$ & $\mathrm{x}$ & $\mathrm{h}$ \\
\hline aproximantes & $\beta_{\mathrm{r}}$ & $\mathrm{r}$ & & & $\mathrm{j}$ & $\mathrm{u}$ & \\
\hline nasales & $\mathrm{m}$ & $\mathrm{n}$ & & & $\mathrm{n}$ & $\mathrm{j}$ & \\
\hline
\end{tabular}

Siete de éstos son marginales. Los fonemas $/ \underline{\mathrm{j}} \underline{\mathrm{c}} \mathrm{c} /$ son demostrablemente derivados de $\mid \mathrm{t}$ p/k x $\mid$ subyacentes mediante el proceso de palatalización, que se discute a continuación. El fonema / $\mathrm{S}$ / es derivado de la $|\mathrm{r}|$ subyacente cuando la vocal que le sigue se elide (véase el apartado 9); al resilabificarse como coda de la sílaba anterior, el rótico se ensordece y se transforma en una fricativa retrofleja. Las velares / u y/ resultan de un proceso de epéntesis en el ambiente $\left|\dot{i} \_V\right|$ que podría considerarse automático pero que antecede a la nasalización (véase el apartado 2) y por ende no puede atribuirse al nivel posléxico. ${ }^{4}$ Finalmente, el fonema $/ \mathrm{h} /$ es de baja frecuencia, alterna con cero y se confunde perceptivamente con $/ \mathrm{x} /$.

El inventario de los fonemas vocálicos se reproduce en la Tabla 2.

Tabla 2. Los fonemas vocálicos del chiquitano migueleño (Nikulin, por aparecer)

\begin{tabular}{|l|c|c|c|}
\hline & anteriores, no redondeadas & no anteriores, no redondeadas & no anteriores, redondeadas \\
\hline altas & $\mathrm{i} \tilde{\mathrm{i}}$ & $\dot{\mathrm{i}} \tilde{\mathrm{i}}$ & $\mathrm{u} \tilde{\mathrm{u}}$ \\
\hline no altas & $\varepsilon \tilde{\varepsilon}$ & $\mathrm{a} \tilde{\mathrm{a}}$ & o õ \\
\hline
\end{tabular}

Las vocales pueden ensordecerse en el margen derecho de una palabra prosódica o entre segmentos sordos (/ßapakitáuro/ [ $\beta_{T}$ a pakị tauro] 'meció'). Secuencias de vocales son tautosilábicas (/taijóuko/ [tai'jouko] 'está volcando') a menos que se encuentren en la posición final de un tema nominal (en este caso se forma un hiato, /pakaúxí/ [paka'ux] 'plátano'). Secuencias de vocales idénticas se pronuncian como vocales largas y jamás se ensordecen (en el margen derecho de una palabra prosódica, pueden realizarse como una vocal corta sonora: /ícoo/ ['ico] 'mi casa', /pakaúkaa/ [paka'uka] 'plátanos'). Además, las vocales cortas tónicas se alargan en el ambiente _ $\{\beta, \mathrm{j}$, u, m, n, $\mathrm{n}\} \mathrm{V \#} \mathrm{(/píno/} \mathrm{['pi:no]}$ 'es lindo'). Las vocales nasales adquieren una fase consonántica (/f́ka?a/ ['ĩnka?] 'ahí') si no están adyacentes a / $/$ o seguidas de /X/ (/ncmana?ú́ko/ [ncmana' ?̦ũko] 'es verdad'), excepto las secuencias tautosilábicas /a $\tilde{\varepsilon}$ o $\tilde{\varepsilon} /$, que jamás adquieren la fase consonántica

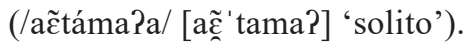

\section{Nasalización}

Todas las variedades del chiquitano presentan un proceso de nasalización ambidireccional a distancia (ver Sans 2011 para el bésiro, Girard 2014 para el chiquitano oriental hablado en San Rafael de Velasco). La nasalización afecta las $\left|\beta_{\tau} \mathrm{r} j\right|$ subyacentes

\footnotetext{
${ }^{4}$ Además, existe un proceso morfofonológico que afecta el sufijo translativo (= predestinativo) $\left|-\beta_{\mathrm{r}}\right|$ y que

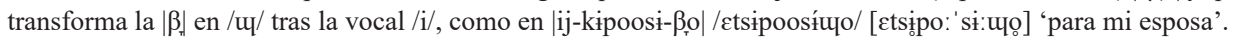


y la $u$ insertada automáticamente en el ambiente $\left|\mathfrak{i}_{-} \mathrm{V}\right|$, transformándolas en $/ \mathrm{m} \mathrm{n} \mathrm{n} \mathrm{y} /$. Prototípicamente, el proceso es desencadenado por (i) vocales nasales, (ii) consonantes nasales y (iii) morfemas que no contienen ningún elemento nasal pero que poseen la respectiva especificación subyacente, que represento como un rasgo flotante $\left.\right|_{[+ \text {nas }]} \mid$ en este trabajo. En 1, se ejemplifica su operación en la flexión. Se muestra que los sufijos flexivos (|-maPa| DIM, |-t $\varepsilon$ ? $\varepsilon_{[+ \text {nas }]} \mid$ 3SG.P) pueden afectar las consonantes de la raíz y de los prefijos (1a-e) y que una raíz puede afectar las consonantes de los sufijos (|-ro|//- $\beta_{\mathrm{r}} \mid$ F. $3 ; 1 \mathrm{f}-\mathrm{i}$ ) y de

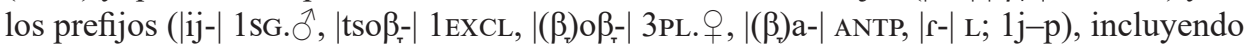
los alomorfos consonánticos de los prefijos subyacentemente vocálicos ( $|\mathrm{o}-|$ IINCL, $\left|\mathrm{i}_{2}-\right|$ M. ̊̄, $\left.\left|\mathrm{i}_{2}\right| \mathrm{INV} ; 1 \mathrm{q}-\mathrm{s}\right)$.

\section{(1) Nasalización en la flexión}

\begin{tabular}{|c|c|c|c|}
\hline a. |kijara-ma?a| & /kinanáma?a/ & 'zorrito. + ' & (cf. /kijaráxi/ 'zorro. ${ }^{\prime}$ ') \\
\hline b. |kurußasi-ma?a| & /kunumasíma?a/ & 'pollito. $q$ ' & (cf. /kurußasíxi/ ‘pollo. $q^{\prime}$ ) \\
\hline c. $\mid$ xu $\boldsymbol{\beta}_{\mathbf{\tau}}$ zjoro-ma?a $\mid$ & /xumenonóma?a/ & 'chilchinita' & (cf. /xußzjoróxi் / 'chilchina') \\
\hline d. |kitsaio-ma?a| & /kitsainóma?a/ & 'nubecita' & (cf. /kitsaiuuóxì ' 'nube') \\
\hline e. $\left|\mathrm{i}_{1}-(\boldsymbol{\beta}) \mathrm{o} \beta_{\tau}-(\boldsymbol{\beta}) \mathrm{a}-\mathrm{t} \varepsilon ? \varepsilon_{[+\mathrm{nas}]}\right|^{5}$ & /nomaté?q/ & 'lo comieron. + ' & (cf. /óußatso/ 'comieron. + ’') \\
\hline f. $\mid \varnothing$-õ-ro $\mid$ & /ốno/ & 'vomitó' & (cf. /st́uro/ 'tronó’) \\
\hline g. $\mid \emptyset$-(呅)a-nitomoko'o-ro $\left.\right|^{6}$ & /manitionokóono/ & 'pagó' & (cf. / ß̦akicóoro/ 'estornudó') \\
\hline h. $\left|\emptyset-(\boldsymbol{\beta}) \mathrm{a}-\mathrm{ki}_{k}^{p} \mathrm{a} \tilde{\varepsilon} \tilde{\varepsilon} \tilde{\varepsilon}-\mathrm{ro}\right|$ & /makiçaź̃̃̃no/ & 'costuró' & (cf. / ßָakapopi?íiro/ 'jugó') \\
\hline i. $\mid(\beta)$ o $\beta_{\tau}-(\boldsymbol{\beta})$ ano- $\boldsymbol{\beta}_{\mathrm{\tau}} \mathrm{o} \mid$ & /oumánomo/ & 'duermen. $\varnothing$ ' & (cf. /orisíuaßָo/ 'está borracho’) \\
\hline j. $|\mathbf{i j}-\tilde{o}-\mathrm{ka}|$ & /jốka/ & 'vomito. §’" & (cf. /jorisiuuáka/ 'estoy borracho') \\
\hline k. $\mid \mathbf{i j}$-apa $a_{[+ \text {nas }]} \mid$ & /jápa/ & 'mi piojo. ${ }^{\lambda}$ ' & (cf. /jikíki/ 'mi uña. ภ’') \\
\hline 1. |ij-aukina?a| & /naukijá?a/ & 'mi seso. ${ }^{\lambda}$ ' & (cf. /jautsási/ ‘mi corazón.ð’’) \\
\hline m. $\left|\mathrm{tso} \boldsymbol{\beta}-\varepsilon_{[+ \text {nas }]}-\mathrm{ka}-\mathrm{ti}\right|$ & /tsomékati/ & 'nos vamos.EXCL' & (cf. /tsußákati/ 'nos fuimos.EXCL') \\
\hline n. $\mid(\boldsymbol{\beta})$ o $\beta_{\text {-pokunũ-Vna } \mid}$ & /mopokunuuná/ & 'contentos. + ' & 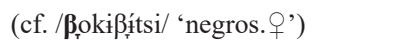 \\
\hline o. $\mid(\boldsymbol{\beta})$ a-pa $\tilde{\imath}-$ ?o $\mid$ & /mapát̃?a/ & 'miente' & (cf. / $\beta$ apáuro/ 'cava') \\
\hline p. $\mid \mathbf{r}$-a-taani $\left.\right|^{7}$ & /natáani/ & 'tu cabeza' & (cf. /rápa/ 'tu brazo') \\
\hline q. $\left|\mathbf{o}-(\beta) a-n i t a{ }^{2} a-k a\right|$ & /manitjáka/ & 'hablamos.INCL' & (cf. /ßָapatáka/ 'mascamos.INCL') \\
\hline r. $\mid \mathbf{i}_{2}$-ato? $\mathbf{1}-\mathbf{x i} \mid$ & /nato?í́ci/ & 'Antonio. $\sigma^{\lambda}$ & (cf. /jaskáte/ 'alcalde. ${ }^{\lambda ’}$ ) \\
\hline s. $\mid \emptyset$-i $\mathbf{i}_{2}-\mathrm{mma}{ }^{2} \mathrm{a}-\mathrm{ta} \mid$ & /namáta/ & 'lo cerró' & (cf. /jokítja/ 'lo cortó') \\
\hline
\end{tabular}

${ }^{5}\left|\mathrm{i}_{1}\right|$ denota una variante de $|\mathrm{i}|$ que se elide delante de vocales, mientras que $\left|\mathrm{i}_{2}\right|$ denota una variante de $|\mathrm{i}|$ que se transforma en $/ \mathrm{j} / \mathrm{o} / \mathrm{n} /$ en el mismo ambiente. Represento como $|(\beta)|$ las instancias de $/ \beta /$ que presentan una alternancia con cero (“/ $\beta$ / móvil”) en determinados ambientes; este fenómeno deberá ser investigado en estudios futuros.

${ }^{6} \mathrm{La}$ notación $\mid \mathrm{V}$ ? $\mathrm{V} \mid$ en la representación subyacente denota las secuencias que se realizan como /V?V/ ante pausa, como $/ \mathrm{VV} /$ ante algunos sufijos (como los que codifican el sujeto de la tercera persona en los verbos finitos) y como $/ \mathrm{V} /$ ante otros sufijos (como los que codifican el sujeto de la primera o de la segunda persona en los verbos finitos). Por ejemplo, la raíz |-nita'a-| 'hablar' se realiza como /-nița?a-/ en /manițá?a/ 'a hablar (nosotros.INCL)', como /-nițaa-/ en /manitiáana/ 'habló' y como /-nița-/ en /manițáka/ 'hablamos.INCL'.

${ }^{7}$ A la diferencia de los demás prefijos, la consonante de ligación $|\mathrm{r}-|$ no se nasaliza al combinarse con material que posee la especificación subyacente de nasalidad pero que no contiene ningún segmento nasal en la

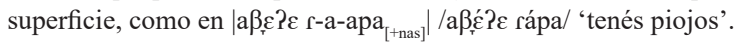


El mismo proceso ocurre en la derivación. En el ejemplo 2a se muestra que un derivado de la raíz |itoßị-| 'noche' sufre la nasalización de la $|\beta|$ se muestra que los sufijos causativizadores $\mid-$ ma $\mid$, $\mid$-na $\mid$ y $\mid-$ ka $^{2} \mathrm{a}_{[+ \text {nas }} \mid$ desencadenan la nasalización de $\left|\beta_{r} r\right|$ en las raíces ( $2 \mathrm{~b}, 2 \mathrm{e}-\mathrm{g}$ ), en el sufijo |-ro| F.3 (2b-d) y en los prefijos, como $|(\beta) a-|$ ANTP $(2 \mathrm{~b}-\mathrm{d})$. En el ejemplo $2 \mathrm{~g}$, la $\mid \beta_{\Downarrow}$ subyacente (presente en la raíz $\mid-$ ta $\left.\beta_{\mathrm{r}} \mathrm{uxi}-\mid\right)$ aparentemente se elidió en el verbo no derivado mediante fusión con $|\mathrm{u}|$, formando una secuencia tautosilábica (/au/), pero no en el causativizado, en que la $|\beta|$ se nasalizó a $/ \mathrm{m} /$.

(2) Nasalización en la derivación ${ }^{8}$
a. /tijomi-çancné-ka/ 'está anocheciendo'
b. / $\varnothing$-ma-taní-ma-na/ 'está quebrando'
(cf. $/ \underline{\mathrm{t}} \mathrm{j} \mathrm{\beta} \mathrm{i} \mathrm{i}-\mathrm{ci} /$ 'noche')
c. /Ø-ma-t fá-ma-na/ 'está invitando bebida'
(cf. /Ø-tári- $\beta_{\mathrm{T}} /$ 'se quebró')
d. /Ø-ma-tisí-na-na/ 'está cortando'
(cf. / $\emptyset$-t $\int a ́-\beta_{\tau} / /$ 'está bebiendo’)
e. /Ø-çunusi-ná-ta/
'lo está/estoy bendiciendo'
(cf. / $\varnothing$-tísi-ro/ 'se cortó')
f. / $\emptyset$-çumuxi-çá-ta/
'lo está/estoy tapando (a alguien)'
(cf. /kurusí-xí/ 'cruz')
g. /Ø-țamuxi-çá-ta/ 'lo está/estoy tapando (un objeto)'
(cf. /Ø-kußúxi-?o/ 'se tapó')
(cf. /Ø-táuxi-?o/ 'está tapado')

No obstante, hay evidencias que muestran que el proceso de la nasalización es sincrónicamente inactivo en el chiquitano migueleño. Como se muestra en el ejemplo 3, las consonantes y las vocales nasales pueden coocurrir con $/ \beta_{\mathrm{r}} \mathrm{r}$ u $\mathrm{u} /$ en una palabra tanto en ítems de origen nativo $(3 \mathrm{a}-\mathrm{h})$ como en préstamos $(3 \mathrm{i}-\mathrm{s})$.

(3) Violación de la "armonía" nasal consonántica
a. /matoríxi//
b. /rominúxi//
'tarechi, loro hablador. + '
c. /kiuyonótso?o/
'apero'
d. /õpakiúóxí/
'es tuerto'
e. /sứtsa $\beta_{\mathrm{T}} \mathrm{O} /$
f. $\tilde{\varepsilon}$ tú $\beta_{\tau} /$
'horcón'
g. /omenetín $\beta_{\mathrm{T}}$ /
'es hediondo'
h. /jakoßóni?i/
‘cuántos', subdialecto de San Juan de Lomerío
'cuántos', subdialecto de San Miguel de Velasco
i. /ánißiso/
j. /kãtaßóxí/
'conmigo'
'mientras, antes de que no', subdialecto de San Juan de Lomerío
(San Miguel de Velasco: /ónimo/)
k. /kaxtrijáanuxi/
'candado'

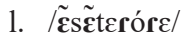
'castellano'
$\mathrm{m} . / \mathrm{t}$ firimóojaxi//
'yesquero, encendedor'
n. /arắkoxi//
'chirimoya'
o. /arißiróne/
'naranja'
p. /rúunici/
'almidón'
q. /remóonici/
'lunes'
r. /ríimaxi//
'limón'
'lima'

${ }^{8}$ En 2b-g, doy formas finitas de verbos causativizados (sujeto de 3sG, voz antipasiva en 2b-d; agente de 3 SG o $1 \mathrm{sG}$, paciente de $3 \mathrm{sG}$, voz inversa en $2 \mathrm{e}-\mathrm{g}$ ). La causativización es un proceso claramente derivacional en el chiquitano migueleño por no ser muy productivo; la escogencia del sufijo causativizador tampoco es predecible. 
s. /maícticu/ 'maestro. + , maestra'

t. /rí̌soxí/ 'lienzo'

En el ejemplo 4 se muestra el comportamiento de las formas flexionadas de algunos verbos prestados del castellano que presentan propriedades "disarmónicas". En todos ellos, la nasalidad se propaga hacia la izquierda, afectando los prefijos (como esperado), pero por alguna razón no lo hace hacia la derecha.

(4) Verbos “disarmónicos"

a. /Ø-ma-kã́sa-ra/ 'está descansando'

b. /Ø-ma-kấta-ra/ 'está cantando'

c. /Ø-ma-pẽsa-ra/ 'está pensando'

d. /n-a-kõpirá-ka/ 'estoy comprando. ${ }^{\lambda}$ '

Otro tema verbal del mismo origen, /-paßz̃̃tcka(a)-/ 'vender', contiene una secuencia que también viola las regularidades discutidas en este apartado (se esperaría que $\mid \beta_{n}$ se nasalizara a $/ \mathrm{m} /$ por la influencia de la vocal $|\tilde{\varepsilon}|)$, pero parece desencadenar la nasalización tanto en los prefijos como en los sufijos: su forma finita de 3sG parece ser /mapaßז̃̃tckáana/ (se la ha observado en el discurso espontáneo, aunque no se encuentra en mi corpus grabado). Analizo esta última forma como $\mid \varnothing-(\boldsymbol{\beta}) \mathrm{a}-\mathrm{pa} \beta \tilde{c} \mathrm{t} \varepsilon-\mathrm{ka}^{2} \mathrm{a}_{[+ \text {nas }]}$-ro| y asumo que el verbo contiene el sufijo causativo $\left|-\mathrm{ka}^{2} \mathrm{a}_{[+ \text {nas }]}\right|$, intrínsecamente nasal, lo que podría explicar por qué este verbo no se comporta como los préstamos análogos en el ejemplo 4.

Finalmente, menciono un fenómeno para el que todavía no se ha podido proponer una descripción completa. Se trata del hecho de que las vocales altas también pueden afectarse por la nasalización, bajo la condición de que sean precedidas por otra vocal o por una /2/. Esto se ejemplifica en el ejemplo 5.

(5) Nasalización de las vocales altas
a. |tsoij-timo?o-ka|
b. $\left|a-i_{2}-t^{2} a_{[+ \text {nas }]}\right|$
/tsoîtjịmóka/
[tsoĩnitffi' mọka]
'estamos sentada/os.EXCL'
c. |ja?i-ma?a|
/aĩtára/
[aĩ' ța?a]
/na?⿱宀ma?a/
[na' ְima?
'a traer (vos u otro)'
d. |taßai-mara $\mid$
/tamaíma?a/
[tama'ínma?]
'niño'
'chichita'

Todavía no ha sido posible determinar si la aplicación de este proceso a las vocales altas está sujeta a alguna restricción adicional.

\section{Africación}

La africación es el nombre que le doy al proceso que transforma las consonantes $|\mathrm{t} \mathrm{k}|$ iniciales de la raíz en $/ \mathrm{t} \mathrm{ts} /$ tras los prefijos |ix-//ij-| 1sG. $/$ /ô, |tsoij-| 1EXCL, ${ }^{9}$ como

${ }^{9}$ Las consonantes finales de estos prefijos se eliden si les sigue una consonante o una /i/, pero se mantienen si les sigue una vocal que no sea /i/ (Nikulin 2019a). En este caso $|\mathrm{x}|$ se realiza como /6/ (ver 4) y $|\mathrm{j}|$ como /j/ (con temas orales) o /n/ (con temas nasales; ver 2): |ix-ikiki|/6ikíki/ [6i'kiki] 'mi uña. $\wp^{\prime}$ ', |ij-ikiki|/jikíki/ [ji'kiki] 'mi

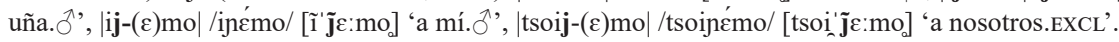


se muestra en el ejemplo 6. El proceso ocurre en todas las variedades documentadas del chiquitano (en bésiro, el resultado de la africación de $|\mathrm{k}|$ no es /ts/ sino /s/, ya que el fonema /ts/ no existe en esa variedad). Como ningún otro morfema demostrablemente termina en $|\mathrm{x}|$ o $|\mathrm{j}|$ (véase el apartado 9), se podría formalizarlo como $|\mathrm{xt} \mathrm{jt}| \rightarrow / \mathrm{t} \mathrm{f} /,|\mathrm{xk} \mathrm{jk}| \rightarrow / \mathrm{ts} /$. Obsérvese que $|\mathrm{k}|$, al transformarse en /ts/, desencadena un proceso de descenso de $|\mathrm{i}| \rightarrow$ $/ \varepsilon /$ en el prefijo (véase el apartado 8).

(6) Africación

\begin{tabular}{|c|c|c|c|}
\hline \multirow{9}{*}{ 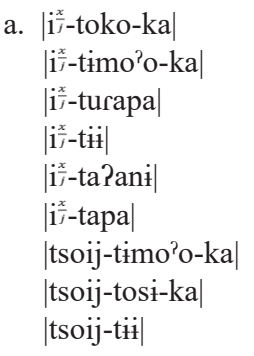 } & /itfokóka/ & [itfo'koka ] & 'estoy bailando' \\
\hline & /itfimóka/ & [itfi'mơka] & 'estoy sentada/o' \\
\hline & /t furápa/ & [t $\int u^{\prime}$ rapa] & 'mi amigo' \\
\hline & /'itfiit/ & ['itfj] & 'mi cuello' \\
\hline & /itfáPani/ & [i'tfaan] & 'mi cabeza' \\
\hline & /ítfapa/ & ['itfapa] & 'mi pierna' \\
\hline & /tsoĩtfïmóka/ & [tsoĩñitfi' mọka] & 'estamos sentada/os.EXCL' \\
\hline & /tsoitfosíka/ & [tsoitfo' sika] & 'tenemos sed.EXCL' \\
\hline & /tsoít/ii/ & [tso'itfi] & 'nuestros cuellos.EXCL' \\
\hline \multirow{6}{*}{ 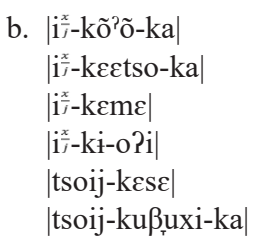 } & /etsốka/ & [ $\varepsilon^{\prime}$ tsõyka] & 'me muero' \\
\hline & /etsectsóka/ & [etse: 'tsōka] & 'tengo vergüenza' \\
\hline & /ع́tseme/ & 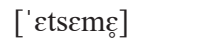 & 'aguja' \\
\hline & /etsiưó?i/ & [Etsi' 'บơ?i] & 'a pisar yo' \\
\hline & /tsoźtsese/ & [tso' $\varepsilon t s \varepsilon s \varepsilon]$ & 'nuestros cuchillos.EXCL' \\
\hline & /tsoetsußuxíça/ & [tsoctsu: 'xiça] & 'nos tapamos.EXCL' \\
\hline
\end{tabular}

Se ha detectado una excepción: la africación no suele afectar el sustantivo /-kícoru/ 'barriga', cuya forma de 1sG ocurre abundantemente como /ikícoru/ [i'kiçorú ' $m i$ barriga' en el habla espontánea. Las y los hablantes han confirmado la gramaticalidad de la forma esperada /etsícoru/, pero las ocurrencias registradas de esta forma se limitan al contexto de la elicitación. Se ha considerado la posibilidad de que la africación en /ikícoru/ [i'kiçor̊u] 'mi barriga' sea bloqueada por el ambiente |i_i| (véase el apartado 4, en que se discute el bloqueo de la palatalización en el mismo ambiente). Sin embargo, la africación afecta regularmente otros temas que se inician con la secuencia |ki|: |i

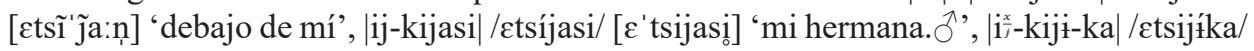
[etsi'jjka] 'nací'. Por ende, el tema/-kícoru/ 'barriga' debe considerarse una verdadera excepción.

\section{Palatalización ${ }^{10}$}

La palatalización es un proceso típico de las lenguas de la región circunchaqueña (González 2014). En chiquitano migueleño, la palatalización que ocurre se trata de una regla que transforma $\left|\mathrm{p} \beta_{\mathrm{r}} \mathrm{m} t \mathrm{kx}\right|$ en $/ \underline{\mathrm{c} j} \underline{\mathrm{t}} \underline{\mathrm{j}} \underline{\mathrm{c}} \boldsymbol{6} / \mathrm{en}$ el ambiente $|\mathrm{i} / \tilde{\mathbf{i}}|$; es decir, la palatalización en esta variedad es progresiva. A pesar de ser un proceso altamente productivo, no se lo

\footnotetext{
${ }^{10}$ Este proceso se discute en más detalle en Nikulin (por aparecer).
} 
puede clasificar como posléxico tanto por la existencia de excepciones léxicas (/ríimaxi/

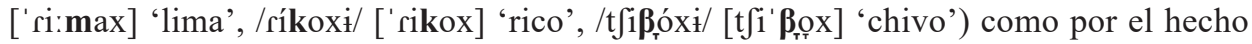
de que la vocal desencadenadora puede elidirse mediante otro proceso morfofonológico

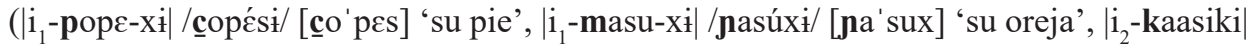

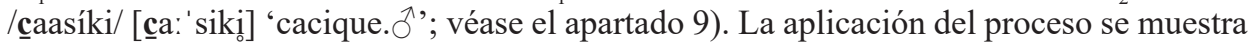
en el ejemplo 7 a continuación.

(7) Palatalización en el chiquitano migueleño
a. $\left|\emptyset-\mathrm{i}_{2}-\mathbf{p} \varepsilon n \varepsilon-\mathrm{ta}\right|$
/c cnéta/
[éc'ncta]
'lo expulsó'
(cf. /péncko/ 'fue expulsado')
b. $\mid \mathrm{i}_{1}-\boldsymbol{\beta}_{\mathrm{T}}$ oto-xi $\mid$
/jotóxi/
[jo' tọx]
'su nalga'
(cf. /aßóto/ 'tu nalga')
c. $\left|\mathrm{i}_{1}-\mathbf{m a s u - x i}\right| \quad /$ nasúxi/
[na'sux]
'su oreja'
(cf. /amásu/ 'tu oreja')
d. |kupiki-ma?a|
/kupikína?a/
[kupi'kĩja?] 'niña'
e. $\left|\emptyset-\mathrm{i}_{2}-\mathrm{ta} \beta \mathrm{ai}-\mathrm{ta}\right|$
/țaßáitita/
[ța' Baitita]
'lo mató'
(cf. /na?ímma?a/ 'niño')
(cf. /tiáta/ 'lo trajo', /ßataßáijo/ 'mató')
f. |a-pitu|
/ápițu/
['apititu] 'tu pipa'
(cf. /kítu/ 'cavando, en la tierra')
g. |tari-kaa|
/tarícaa/
[ta' rica]
'petos. + '
(cf. /anenćkaa/ 'días')
h. $\left|\mathbf{i}_{1}-\mathbf{k} \varepsilon m \varepsilon-\mathbf{x} \dot{\mid}\right|$
/ćcmési/
[c c $\left.\varepsilon^{\prime} \mathrm{m} \varepsilon \mathrm{s}\right]$
'su aguja'
/tomíco/
[to' mĩn_co]
'domingo'
(cf. /kemésì ' 'aguja')
j. $\mid i_{2}$-xara-xi $\mid$
/caráxí/
[ca'rax]
'cruceño. ${ }^{\lambda}$ '
(cf. /nốka/ 'vomité. ${ }^{\lambda}$ ')
k. $\mid i_{i}^{\frac{x}{i}}$-xina-ka $\mid$
/icináka/
1. |pa?ĩ-xí|
[icĩ'jaka]
'quiero'
(cf. /xaráxí/ 'cruceño.早')
[pa' P̃íc]
(cf. /axijáka/ 'querés')
(cf. /pa?́́xi/ 'mujer')

La palatalización de $\left|\mathrm{p} \beta_{\mathrm{\tau}} \mathrm{m} \mathrm{t} \mathrm{k}\right|$ (pero no la de $|\mathrm{x}|, 7 \mathrm{k}-1$ ) sufre un bloqueo en el ambiente $\left|\mathrm{i} / \tilde{1} \_\mathrm{i} / \mathrm{i}\right|$, como se muestra en el ejemplo 8.

(8) Bloqueo de la palatalización en el chiquitano migueleño
a. $\mid \mathrm{i}_{2}$-piripi $\mid$
/pirípi/
[pi'ripi]
'Felipe. ${ }^{\lambda}$ '
b. |tipi-xi|
/tipíci/
[ti'pic]
'hormiga. ${ }^{\circ}$ '
c. |kißitfo-xi $\mid$
/kißitfóxí/
[kißit t tọx]
'camote'
d. $\mid \emptyset$ - $(\beta) a-\tilde{\varepsilon}$ tonimi-?o $\mid$
[mačto' nimijio]
e. $\mid i_{2}$-mikora-xí|
/maz̃toními?o/
[miço' rax]
f. $|o(\beta) i-t i p i|$
/miçoráxi/
[oi'ti?i]
g. $\left|i_{1}-\mathbf{k i - x i}\right|$
/oití?i/
[i'kic]
'se lava las manos'
'Nicolás. $ð$ '
'por él. ${ }^{1}$ '
/ikíci/
[kupi'kic]
'su pluma, su vello'
/kupikíci/
'joven (mujer)'

El reconocimiento de la palatalización progresiva como un proceso activo implica que los segmentos / $\underline{\mathrm{t}} \underline{\mathrm{j}} \mathrm{b}$, contrastivos en el nivel fonológico, no son imprescindibles para la representación subyacente: se los puede considerar derivados de $|\mathrm{p} / \mathrm{k} \mathrm{t} \mathrm{x}|$ en todas las instancias, ya que / $\underline{\mathrm{t}}$ $/$ jamás ocurren tras vocales que no sean /i $\tilde{\mathrm{i}} /$. En cambio, no todas las instancias de $/ \mathrm{j} \mathrm{j} /$ son derivadas, ya que hay múltiples palabras en que estos segmentos aparecen en ambientes en que no se pueden considerar derivados: /motojo?ćsi/ 'motoyoé.

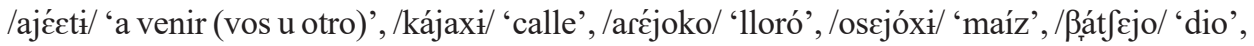
/kitojî́xixi/ 'seboí. o'’ (subdialecto de San Miguel), /(h)áno/ 'ustedes', /kóojo/ 'se murió', /sajoráxi// 'señora', /manaxíci/ 'manechi. o+', /عtsencnáka/ 'estoy sudando', /anćsi// 'carne’, /makojóxi்/ 'macono. 
Obsérvese que en algunos casos no hay evidencias internas al chiquitano migueleño que indiquen si una determinada instancia de /ç/ debe derivarse de $|\mathrm{p}|$ o de $|\mathrm{k}|$. Para estos ítems se postula el archifonema $\left|\frac{p}{k}\right|:\left|-k i i_{k}^{p} \mathrm{a} \tilde{\varepsilon}^{?} \tilde{\varepsilon}-\right| /-k i \underline{c} a \tilde{\varepsilon}(\tilde{\varepsilon})-/$ 'costurar',

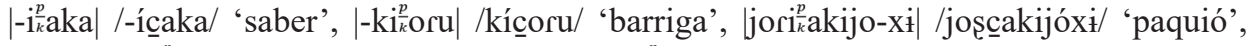

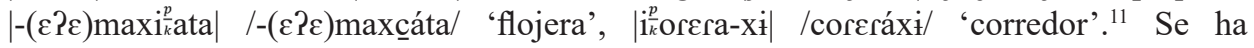
planteado adoptar la misma estrategia para las instancias de /j/ y /n/ precedidas de /i/ y

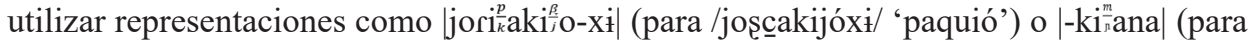
/-kinána/ 'debajo de'), pero en este caso el uso de archifonemas no conllevaría ventajas analíticas, ya que las $|\mathrm{j} \mathrm{j}|$ subyacentes son parte del inventario.

\section{Minimalidad de los temas nominales y adposicionales}

Hablando propiamente, no se trata de un proceso morfofonológico sino de una restricción al tamaño mínimo de los temas nominales y adposicionales en el chiquitano migueleño. Más específicamente, la combinación del tema nominal o adposicional con los eventuales prefijos posesivos debe contener al menos dos moras en la superficie (sin que se cuenten los eventuales sufijos). Ello contrasta con la situación del bésiro, en que no existe tal restricción. A los temas monomoraicos del bésiro les corresponden temas bimoraicos en el chiquitano migueleño, con una secuencia del tipo/V?V/ correspondiendo a una vocal corta en el bésiro, como se muestra en el ejemplo 9.

(9) Correspondencia entre migueleño /VPV/ y bésiro /V/

\begin{tabular}{|c|c|c|c|c|}
\hline mig & & bési & & \\
\hline a. /ta?á-xí/ & [ta' ${ }^{\prime}$ ax $]$ & /tá-sì) & ['tas] & 'llu \\
\hline b. /suPú-xi/ & [su' $\underset{T}{\mathrm{~T}} \mathrm{ux}$ ] & /sú-șìt & ['sus] & 'sol' \\
\hline . /kìín-xí/ & [ki' & /kí-sì / & ['kis] & 'tiern \\
\hline /pع?ć-sí/ & {$\left[\mathrm{p} \varepsilon^{\prime}{ }_{T}^{\prime} \varepsilon \mathrm{s}\right]$} & /pé-se/ & ['pes] & 'fueg \\
\hline
\end{tabular}

Hay una aparente excepción a esta regularidad. Me refiero a los temas como /-su/ 'cara, rostro', /-pa/ 'brazo, ala', /-ki/ 'vello, pluma', /-ta/ 'en la cabeza de' (y potencialmente algunos otros). Obsérvese, sin embargo, que esos temas jamás ocurren sin un prefijo, ya que la expresión del poseedor/complemento en ellos es obligatoria. Por esa razón, no hay motivos para que sus vocales subyacentes se conviertan en una secuencia del tipo/V?V/: el prefijo provee una mora adicional, haciendo que el tema flexionado satisfaga la condición de minimalidad sin que ningún otro ajuste sea necesario. Esto se muestra en el ejemplo 10.

${ }^{11}$ En todos estos casos, se puede recuperar la calidad original de la consonante acudiendo a datos comparativos, pero las evidencias de este tipo son irrelevantes para un análisis sincrónico. Los cognados de las formas citadas en el bésiro son como sigue: /-kipiẽ-/ 'costurar', /-ipaka/ 'saber', /-kiporo/ 'barriga', /n-ori-pakio-si// 'paquió' (Sans 2010: 160), /-emaşikata/ 'flojera'; la última forma es un préstamo antiguo del castellano (corredor /koredor/ [kore' 
(10) Temas de estructura /-CV/ en el chiquitano migueleño
a. $/ \varepsilon^{\prime}$-su/ ['esul $] \quad$ 'mi cara, mi rostro'
b. /á-pa/ ['apa] 'tu brazo'
c. /jo-kí-ci/ [jo'kic] 'sus plumas (de ellos, $q$ )'
d. /á-ta/ ['atå] 'en tu cabeza; sobre vos'

Una peculiaridad de los temas de estructura /-CV/ se hace evidente al considerarse sus formas flexionadas para la tercera persona del singular. El prefijo de 3sG, $\left|i_{1}\right| \mid$, se realiza casi siempre como la palatalización de la consonante inicial del tema o como cero, si el segmento inicial es una consonante no palatalizable o una vocal. La palatalización es una pista que permite suponer que el prefijo de 3 sG es un prefijo segmental $\left|i_{1}-\right|$ que se elide sin importar si hubo palatalización o no del segmento inicial (salvo que en un caso no deja ningún vestigio). Sin embargo, al prefijarse a un tema monomoraico, $\left|i_{1}-\right|$ no sufre elisión, como se muestra en el ejemplo 11, lo que pone en evidencia su carácter segmental.

(11) Temas de estructura /-CV/ flexionados para 3SG
a. $\mid \mathbf{i}_{1}-$ su-xi $\mid$
/Esúxí/ [ $\quad\left[\varepsilon^{\prime} \operatorname{sux}\right]$
'su cara, su rostro'
b. $\mid i_{1}-$ pa-xi $\mid$
/ićáxì/ [i'çax]
'su brazo, su ala'
c. $\left|i_{1}-k i-x i\right|$
/ikíci/ [i'kic]
d. $\mid i_{1}-$ ta $\mid$
/ítja/ ['itija]
'su vello, su pluma'
'en la cabeza de'

compárese con:

\begin{tabular}{|c|c|c|c|}
\hline e. $\mid \mathbf{i}_{1}$-suto-xí $\mid$ & /sutóxí/ & [su'tox] & 'su ojo' \\
\hline f. $\mid i_{1}-$ pakã?ã-xí| & /çakã?ã́xi்/ & [cakã' ?̦ãx] & 'su hígado' \\
\hline g. $\mid \mathbf{i}_{1}-$ poo-xí $\mid$ & /çoóxí/ & ['co: $\underline{0}: x]$ & 'su casa' \\
\hline h. $\left|\mathbf{i}_{1}-\mathrm{t} \dot{\mathbf{i}}-\mathbf{x} \dot{\mathbf{x}}\right|$ & /țííxít & {$\left[{ }^{\prime} \underline{\mathrm{t}} \mathrm{t}: \mathrm{x}\right]$} & 'su cuello' \\
\hline i. $\mid \mathrm{i}_{1}-\mathrm{si}$ i $\mid$ & /sí?i/ & ['si?i] & 'tras, por, a las' \\
\hline
\end{tabular}

La relación estrecha entre las distintas clases de temas según necesiten obligatoriamente un prefijo de poseedor y la existencia o no de temas monomoraicos en cada clase puede ser capturada postulando una estructura subyacente $|\mathrm{CV}|$ para los temas en (9) y una regla de formación de pie mínimo, que transformaría temas subyacentemente monomoraicos no poseídos (por ejemplo, |ta-| 'lluvia', |su-| 'sol',

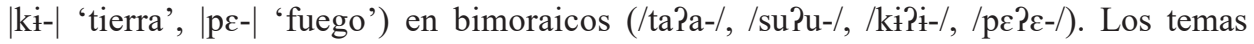
subyacentemente monomoraicos que siempre son poseídos conservarían su estructura subyacente, pero se bloquearía la elisión de la vocal contenida en $\left|i_{1}-\right|$ SSG (un proceso que ocurre en todos los demás temas) para evitar violar la restricción de la minimalidad. ${ }^{12}$

${ }^{12}$ Un proceso casi idéntico afecta los temas nominales y verbales del maxacalí, una lengua macro-ye hablada en la periferia oriental de la zona macro-ye (en el nordeste de Minas Gerais, Brasil). Según Silva (2020a, b), los temas subyacentemente monomoraicos del maxacalí pueden pasar por un proceso de "alargamiento" (V $\rightarrow \mathrm{VhV} \mathrm{o} \mathrm{VPV),} \mathrm{que} \mathrm{ocurre} \mathrm{solamente} \mathrm{si} \mathrm{el} \mathrm{tema} \mathrm{no} \mathrm{posee} \mathrm{un} \mathrm{argumento} \mathrm{interno} \mathrm{y} \mathrm{tampoco} \mathrm{es} \mathrm{el} \mathrm{argumento}$ interno de algún otro tema. Por esa razón, los temas cuyo argumento siempre se expresa poseen solamente un alomorfo monomoraico (por ejemplo, $|\mathrm{ktuk}|$ /ktuk/ 'hijo', que jamás ocurre sin un poseedor). Los demás temas 


\section{Armonía vocálica en afijos y clíticos específicos}

Algunos morfemas funcionales del chiquitano presentan patrones idiosincráticos de armonía vocálica, que se detallan a continuación.

El primer grupo incluye algunos sufijos que contienen la vocal $|\mathrm{o}|$, incluyendo $\mid-$-ro $\mid$ F.3, |-Po| F.3, el sufijo de nomina instrumenti |-to| y el predestinativo/translativo $\left|-\beta_{\mathrm{r}}\right|$. Normalmente la vocal de estos sufijos se realiza como /o/. En el ejemplo 12 se muestra que ésta cambia a /a/ si la sílaba final del tema al que se le agrega el sufijo contiene una /a/ (y aparentemente /ã̃/, como en 12f).

(12) Armonización $|\mathrm{a} \ldots \mathrm{o}| \rightarrow / \mathrm{a} \ldots \mathrm{a} /{ }^{13}$
a. $\mid \emptyset$-arapa-ro $\mid$
/arápara/
'se derramó'
(cf. / ßarákicto/ 'se echó')
b. $\mid \varnothing-(\beta)$ a-pasc $\varepsilon^{2} a-r o \mid$
/ß̧араsćara/
'visitó, paseó'
(cf. / ßapakitáuro/ 'cosechó')
c. $\left|\emptyset-(\beta) a-n_{i}{ }^{2} a-r \mathbf{0}\right|$
/manituáana/
'habló'
(cf. /maumécno/ 'tejió')
d. $\mid \varnothing$ - $(\beta)$ a-tisi-na-ro $\mid$
'cortó'
(cf. /maũxókono/ 'se enfermó')
e. $\mid(\beta) o(\beta)$-pasa-?o|
/matisínana/
'pasaron. ${ }^{\prime}$ '
(cf. /ßrupáki?o/ 'se cayeron. $\varnothing$ ')
f. $\mid \emptyset-(\beta) a-p a \tilde{t}-\{\mathbf{o} \mid$
'mintió'
g. $\left|(\beta) a-a^{2} a_{[+n a s]}-t \mathbf{0}-\mathbf{x} \dot{\mathbf{x}}\right|$
/mapát̃?a/
/maatáxí/
'anzuelo'
(cf. /́́rî̀/ 'se bautizó, se llama')
h. $|(\beta) a-i n a m a-t o-x \dot{i}|$
'urupé'
i. $|(\beta) a-p \varepsilon s a-t o-x i|$
/mainamatáxi/
'pesas'
j. $\left|\mathrm{i}-\mathrm{t} \int \mathrm{a}-\beta_{\mathrm{T}}\right|$
/mapesatáxi/
/itfáßa/
'voy a tomar'
(cf. / ßautakitióxí/ 'piedra de afilar') $)^{14}$
(cf. /masuunkotóxi/ 'escoba')
(cf. /mapunotóxí/ 'flauta')
(cf. /icóoß̦o/ 'mi futura casa')

El proceso no se aplica a varios otros sufijos con función idéntica, como $|-\beta \mathrm{o}|$ F.3

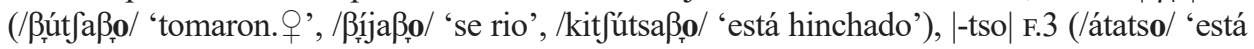
lleno', /óußatso/ 'comieron. 9 ') y el sufijo de nomina instrumenti |-po| (/tfapóxí/ 'vaso'). Por ello, concluyo que la armonización $|\mathrm{a} \ldots \mathrm{o}| \rightarrow / \mathrm{a} \ldots \mathrm{a} /$ es un fenómeno profundamente morfofonologizado.

Otro tipo de armonización, compartido por el migueleño con todas las demás variedades del chiquitano (cf. Krüsi \& Krüsi 1978), afecta el nominalizador |-ki|, cuya vocal normalmente se transforma en una copia del núcleo de la sílaba anterior (si ésta es nasal, se transforma en su equivalente oral), como se muestra en el ejemplo 13. Hay

subyacentemente monomoraicos presentan una alternancia entre dos formas (por ejemplo, $|\mathrm{kut}| \rightarrow / \mathrm{kuhut} / \sim / \mathrm{kut} /$ 'yuca'). Otra lengua que presenta un fenómeno análogo es el guaraní occidental (chiriguano), en que la vocal

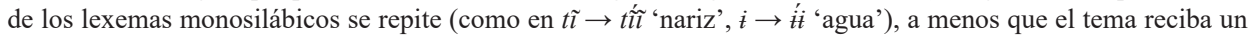
prefijo, como en nét i 'tu nariz', o un sufijo, como en ípe 'en el agua' (Dietrich, 1986, p. 57). Un(a) evaluador(a) anónimo/a sugiere que se podría tratar de un rasgo areal.

${ }^{13}$ Un fenómeno análogo ocurre también en las demás variedades del chiquitano, pero es oportuno observar que en el bésiro (y potencialmente en algunas otras variedades) $|\mathrm{o}|$ no se asimila únicamente a la /a/ sino también a la /u/ precedente, lo que jamás ocurre en el chiquitano migueleño: bésiro /ßapakitáuru/ 'cosecha maíz', /kusíuru/ 'tiene fuerza', /takírusu/ 'se acabó' (cf. migueleño / ß̦apakitáuro/, /kosíuro/, /takírutso/).

${ }^{14}$ Admito la posibilidad de que la representación correcta de este ítem sea /ßָautakukițóxí/, ya que se trata de una derivación de la raíz /-utakuki-/ (/ - $\beta_{\tau}$ a-utakúki-?o/ 'afiló'). Dispongo de apenas una instancia de esta palabra en mi corpus, en la que la esperada vocal /u/ no es audible; es posible que se haya ensordecido o elidido en el ambiente átono entre segmentos sordos. La correcta representación del ítem para 'piedra de afilar' deberá confirmarse en trabajos de campo futuros. De todos modos, la cuestión es irrelevante para el fenómeno presentado en este apartado. 
dos situaciones excepcionales: (i) si la sílaba anterior contiene /E/ (no se han registrado ejemplos con / $\tilde{\varepsilon} /$ ), el sufijo se realiza simplemente como /-ki/ (13g); (ii) si la sílaba anterior

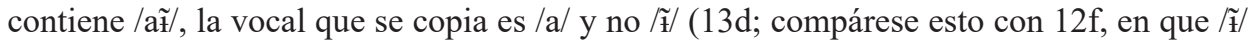
en una secuencia tautosilábica es igualmente transparente). ${ }^{15}$

(13) Armonización $|\mathrm{V} \ldots \mathrm{i}| \rightarrow / \mathrm{V} \ldots \mathrm{V} /$

\begin{tabular}{|c|c|c|c|}
\hline a. $\mid \mathbf{i}$-takisirit-ki-xí| & /țjakisirikikíxí/ & [țakisisiri'kix] & 'su sufrimiento' \\
\hline b. $\left|\mathbf{i}_{1}-(\beta) a-u-k \mathbf{i}-\mathbf{x} \mathbf{i}\right|$ & /jaukúxí/ & [jaun'kux] & 'su ladrar' \\
\hline c. $\left|\mathbf{i}_{1}-\left(\beta_{\tau}\right) a-n i t a ? a-k \mathbf{i}-\mathbf{x}\right|$ & /nanițakáxí/ & [nantija'kax] & 'su voz' \\
\hline d. $\left|\mathbf{i}_{1}-(\beta) a-p a \tilde{a}-k \mathbf{i}-\mathbf{x} \dot{\mid}\right|$ & /napaĩkáxì/ & [napa:̃ 'kax] & 'su mentira' \\
\hline e. $\mid\left(\beta_{r}\right)$ a-kixo?o-ki-xi $\mid$ & /ß̧akicokóxi̇/ & 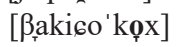 & 'estornudo' \\
\hline f. $\left|k \tilde{o}^{2} \tilde{o}-k \mathbf{i}-x \dot{i}\right|$ & /kõkóxí/ & [kõy'kox] & 'muerte' \\
\hline g. $\left|(\beta) a-t \int \varepsilon-k \mathbf{i}-x \dot{i}\right|$ & /ßat $\int \varepsilon k i ́ c i /$ & [ $\beta_{T}$ at $\int \varepsilon^{\prime}$ kic] & 'golpe' \\
\hline h. $\left|i_{1}-p \varepsilon \varepsilon-k \mathbf{i}-x \mathbf{i}\right|$ & /çcعkíci/ & [çe:'ki6] & 'su calor' \\
\hline
\end{tabular}

El tercer tipo de armonización afecta los sufijos $\mid$-xi| (que ocurre en los sustantivos sin un poseedor referencial, en su forma del singular no diminutiva), |-pi| (segundo elemento del circunfijo negativo; dubitativo) y |-ti| (direccional centrífugo/centrípeto). La vocal de estos sufijos se realiza normalmente como /it/, pero se anterioriza a /i/ si el núcleo de la sílaba final del tema es /i ĩ/, como se muestra en el ejemplo 14 .

(14) Armonización $\mid \mathrm{i} \ldots$...i $\mid \rightarrow / \mathrm{i} \ldots \mathrm{i} /$

\begin{tabular}{|c|c|c|c|}
\hline a. $\mid \mathrm{t} \int \mathrm{i}-\varnothing$-ura-p-o?i-pi $\mid$ & /t fîurapóPipi/ & [t]iurra' pợipi] & 'no avisó' \\
\hline 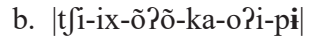 & /t tîicõ?õkóPipi/ & 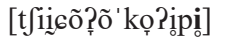 & 'no oigo $(q)$ ' \\
\hline c. $\mid t \int \mathrm{i}-\mathrm{i}^{\frac{x}{i}}-$ suputa-ka-a?i-pi $\mid$ & /t $\int \varepsilon$ ?EsuputakáPipi/ & [t $\int \varepsilon_{\sim}$ supuputa ka?ipipi] & 'no conozco' \\
\hline d. $\mid$ tsai-pi $\mid$ & /tsáipi/ & ['tsaipi] & 'casi' \\
\hline e. $\mid \emptyset$-tsiro-ti?i-ti $\mid$ & /tsirotíPiti/ & [tsiro' tiiti] & 'se va $(\overbrace{}^{\lambda})$ ' \\
\hline f. $\left|i_{1}-t \int i k i-x \mathbf{i}\right|$ & /tfikíci/ & [tfi'kic(i)] & 'su huevo' \\
\hline g. $|\operatorname{ari}-\mathbf{x} \mathbf{i}|$ & /aríci// & [a'ric(i)] & ‘ají’ \\
\hline
\end{tabular}

La anteriorización en los sufijos |-pi| y |-ti| podría atribuirse a la necesidad de preservar la calidad de sus consonantes iniciales: éstas deberían palatalizarse en el ambiente $\left|\mathrm{i} / \tilde{I}_{-} \mathrm{V}\right|$, pero la palatalización se bloquea si la vocal que les sigue es $|\mathrm{i}|$. Sin embargo, la explicación no funciona en el caso del sufijo |-xí|, cuya consonante tiene la particularidad de palatalizarse incluso en el ambiente $\left|\mathrm{i} / \tilde{1}_{-} \mathrm{i}\right|$.

Finalmente, presento dos procesos de armonización regresiva translaríngea, ambos opcionales y típicos del habla rápida. El primero afecta el prefijo $|\mathrm{t} f \mathrm{i}-|$ (en el subdialecto de San Miguel) o $|\mathrm{t} f \mathrm{i}-|$ (en el subdialecto de San Juan), que puede analizarse como negativo (cuando forma un circunfijo con |-pi|) o como condicional. Si la forma a la que se agrega este prefijo empieza por una vocal, el encuentro vocálico puede resolverse mediante la elisión de la vocal del prefijo (/óxina/ 'es bueno' $\rightarrow$ /t foxinaṕ́rí/ 'no es bueno') o mediante

${ }^{15}$ Un(a) evaluador(a) anónimo/a observa que se podría describir la armonización de $\mid$-ki| como una asimilación de una vocal alta anterior a una vocal no anterior, lo que explicaría por qué el proceso no ocurre tras /ع/. 
la epéntesis de /P/ (/icáka/ 'comí' $\rightarrow$ /t firicákapi/ 'no he comido'). En este último caso la vocal del prefijo puede asimilarse a la vocal inicial del tema en el habla rápida, como se muestra en el ejemplo 15.

(15) Armonización translaríngea de $|\mathrm{i} / \mathrm{i} \ldots \mathrm{V}| \rightarrow / \mathrm{V} \ldots \mathrm{V} /$
a. /tfiresuputaká?ipi/
$\sim / \mathrm{t} \int \varepsilon$ ? ssuputaká?ipi/
[t]i£suputa'kaPipi] [no conozco'
$\sim\left[\mathrm{t} \int \varepsilon_{\sim} \operatorname{suputa}_{\circ} \mathrm{kaPipi}_{\mathrm{o}}\right]$

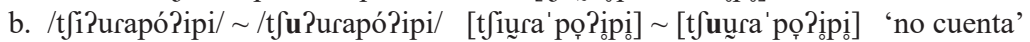 $\sim / \mathrm{t}$ furapó

Algo similar afecta algunos demostrativos que terminan en $\mid-\mathrm{a}$ ?a $\mid$ : $\mid \beta_{\mathrm{\tau}} \mathrm{P}$ ?a $\mid$ 'estos', |na?a $\mid$

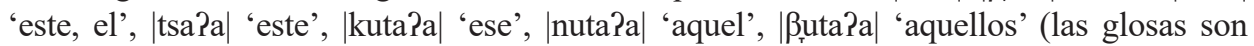
aproximadas, ya que todavía no se ha podido determinar todas las oposiciones relevantes para la deixis). Estos elementos integran el mismo grupo de acento que el sustantivo siguiente, motivo por el que los describo como clíticos. Si la palabra que les sigue empieza por una vocal, la vocal final del demostrativo se elimina (/ $\beta_{T}$ ? utsaitáiki/ 'estos nuestros.INCL

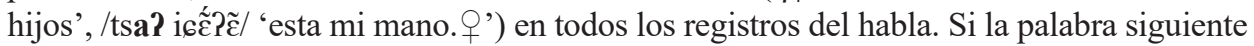
empieza por una /o/, la |a| del demostrativo puede opcionalmente asimilarse a /o/ en el habla rápida. El ejemplo 16 contiene datos que provienen del habla espontánea.

(16) Armonización de $\mid \mathrm{a}$ ?a\#o $\mid \rightarrow / 0$ ?o/
a. /na?a/+/oscjóxí/ $\rightarrow \quad$ /no? oscjóxí/
[noose'jọ] 'el maíz'
b. / $\beta_{\tau} \mathrm{aPa} /+$ /oni?́́kaa/ $\rightarrow / \beta_{\mathrm{T}}$ ? oni?́́kaa/
[ßоooji' 'ika]
'estos hombres. + '
c. /tsa?a/ +/ótu/ $\rightarrow$ /tso? ótu/
[tso' ?ִotulu]
'esta tu lengua'
d. /kuta?a/ + /oni?́xíl $\rightarrow$ /kuto? oni?́xi/
[kutooni' ?̦ix]
'ese hombre. ' '
e. /nuta?a/+/oni?́xi/ $\rightarrow$ /nuto? oni?́xi/
[nutooni'? ?̦ix]
'aquel hombre. $\varnothing$ '

La opcionalidad del proceso se demuestra por las ocurrencias de ejemplos como /na? oscjóxi/ 'el maíz', /na? okóxi/ 'el joco'. En efecto, uno de mis consultantes (fallecido en 2019) caracterizaba las formas armonizadas como "incorrectas", aunque se las constató en su habla fluida.

\section{Elevación de $|\mathbf{0}|$ a /u/}

La elevación de $|\mathrm{o}| \mathrm{a} / \mathrm{u} /$ delante de una sílaba que contiene /a/ se ha documentado en todas las variedades del chiquitano. En la variedad migueleña, el proceso ocurre regularmente, sin ninguna excepción, en los prefijos |o-| 1 INCL, |tso $\beta_{\mathrm{r}} \mid$ 1 $\mathrm{EXCL},\left|\left(\beta_{\mathrm{r}}\right) \mathrm{o} \beta_{\mathrm{r}}\right|$ 3PL. + , |o-| NHA. $\hat{0}$, como se muestra en el ejemplo 17. 
(17) Elevación de $|\mathrm{o}|$ en prefijos

\begin{tabular}{|c|c|c|c|}
\hline a. $\mid$ o-ts-axłpcku?u| & /utsáxpeku?u/ & [u'tsaxpeku?] & 'entre nosotros.INCL' \\
\hline b. $\mid \mathbf{o}-\mathrm{a}$ ?a $\mid$ & /úßa?a/ & ['ußa? ] & 'a comer nosotros.INCL' \\
\hline c. |o-pai $\mid$ & /úpai/ & ['upai] & 'nuestra.INCL leche' \\
\hline d. $|\operatorname{tso} \beta-a-k a-t i|$ & /tsußákati/ & [tsu' $\left.\beta_{\tau}^{\text {wakati }}\right]$ & 'nos fuimos.EXCL' \\
\hline e. $\mid \operatorname{tso} \beta_{\tau}-\left(\beta_{\tau}\right)$ a-topi $\mid$ & $/$ tsupátopi/16 & [tsu' patopi] & 'a bañarnos.EXCL' \\
\hline f. $\mid \mathrm{i}_{1}-(\beta) \mathbf{o} \beta_{T}-\beta \mathrm{a}$ ?u-xi $\mid$ & /jupa?úxí/ & [jupa'?ux] & 'sus animales (de ellos,, )' \\
\hline g. $\mid(\beta)$ o $\beta$-tatsu $\beta$ a-tso?o $\mid$ & / $\beta_{\tau} u t a t s u ́ \beta$ atso?o/ & [ßita'tsuwatso?] & 'son redondos. + ' \\
\hline h. $\mid \mathbf{o}$-tari-xí| & /utaríci/ & [uta'ric] & 'peto. $\sigma^{\text {' }}$ \\
\hline $\mid \mathbf{0}$-mas $\varepsilon$-xí| & /umasćsì/ & [uma'ses] & 'masi. $\delta^{\lambda}$ \\
\hline
\end{tabular}

Sin embargo, el proceso no es totalmente automático, ya que las secuencias del tipo /o...a/ no son prohibidas en el chiquitano migueleño: /poókaa/ ['pợa] 'casas', /tsomá/ [tso' ma] 'para que no; ProH', /kóta?a/ ['kọtaa] 'dónde'.

Un proceso fonológico casi idéntico se ha atestiguado en el guarasugwe, una lengua tupí-guaraní hablada al norte de la Chiquitanía (Ramirez et al. 2017). Las dos lenguas comparten también un proceso de palatalización progresiva, que se discutió para el chiquitano migueleño en 4. Es probable que el guarasugwe y el chiquitano hayan estado en contacto en el pasado y que algunos procesos fonológicos se hayan difundido del chiquitano al guarasugwe (Nikulin 2019c: 9-10).

\section{Descenso de $|\mathrm{i}| \mathrm{a} / \varepsilon /$}

El descenso de $|\mathrm{i}|$ a $/ \varepsilon /$ delante de /s ts/ ocurre regularmente, sin ninguna excepción, en los prefijos |ix-|/|ij-| 1sG. $/ \widehat{\partial}^{\lambda}$, |tsoij-| 1EXCL, |i ${ }_{1} \mid$ 3SG, como se muestra en el ejemplo 18. $\mathrm{La} / \mathrm{ts} /$ derivada de $|\mathrm{k}|$ mediante la regla de africación (apartado 3) también desencadena el proceso.

${ }^{16}$ Este ejemplo y el ejemplo siguiente instancian el proceso $|\beta \beta| \rightarrow / \mathrm{p} /$, que ocurre sobre todo cuando a uno

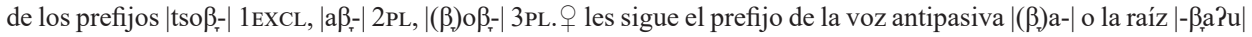
'animal.PL', generando las secuencias /tsupa.../, /apa.../,/(u)pa.../. La fortición de $|\beta|$ a $/ \mathrm{p} /$ en estos prefijos se observa también si el morfema siguiente se inicia por /i/, generando las secuencias /tsopi.../,/api.../,/opi.../. En efecto, es probable que al menos algunas instancias de $\mid \beta_{n}$ en chiquitano provengan de una lenición histórica de * $p$ en el ambiente intervocálico; la secuencia *pp no habría sufrido la lenición, resultando en $\left|\beta \beta_{n}\right| \mathrm{p} /$. Este supuesto cambio podría estar involucrado, además, en la relación entre los temas /pitsi-/ 'negro (persona)' y /-kißítsi/ 'negro (color)': en las fuentes coloniales (Adam \& Henry 1880: 136) se han atestiguado separadamente la raíz 〈-bizi〉 'negro' y el prefijo «quì-> (de semántica desconocida, usado con los términos de color). De esta manera, la fosilización de la raíz /-kiß̣̂́tsi/ podría ser un desarrollo reciente (otro tema afectado por este tipo de fosilización sería /-kitúriki/ 'rojo', cf. «-turiqui〉 en Adam \& Henry 1880: 136). 
(18) Descenso de $|\mathrm{i}|$ en prefijos

\begin{tabular}{|c|c|c|c|}
\hline a. $\left|\mathbf{i}_{\overline{\mathbf{i}}}^{\frac{x}{-}} \mathrm{k} \varepsilon \mathrm{m} \varepsilon\right|$ & /ع́tseme/ & 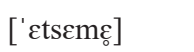 & 'mi aguja' \\
\hline b. $\mid \mathbf{i}^{\frac{x}{\gamma}}$-kamisa $\mid$ & /etsamísa/ & [etsa'misa] & 'mi camisa' \\
\hline c. $\left|\mathbf{i}_{\bar{i}}^{\frac{x}{i}-\mathrm{ko}} \tilde{\mathrm{o}}^{\mathrm{O}} \tilde{\mathrm{o}}-\mathrm{ka}\right|$ & /etsốka/ & [ $\varepsilon^{\prime}$ tsõykạ] & 'me muero' \\
\hline d. $\mid \mathbf{i}_{i-\operatorname{si}}^{\frac{x}{i}} \beta_{\mathrm{T}}$ ori-ka| & /esißß̦oríça/ & [Esißot rica] & 'vivo' \\
\hline e. $\left|\mathbf{i}^{\frac{x}{\gamma}}-\mathrm{su}\right|$ & /'́su/ & ['esu] & 'mi cara, mi rostro' \\
\hline f. $\mid \mathbf{i}^{x}$-ts-o?o $\mid$ & /عtsó?o/ & [E'tsơ?o] & 'mi diente' \\
\hline g. $\mid \mathbf{i}_{\bar{y}}^{x}$-ts-ai $\mid$ & /'́tsai/ & ['Etsai] & 'mi hijo' \\
\hline h. $\mid$ tsoij-kese $\mid$ & /tsoztsese/ & [tso' $\varepsilon \operatorname{ts} \varepsilon s \varepsilon]$ & 'nuestros.EXCL cuchillos' \\
\hline i. |tsoij-sut $\int \varepsilon-k a \mid$ & /tsoesutfćka/ & [tsoesu't $\left.\int \varepsilon k a\right]$ & 'estamos tristes.EXCL' \\
\hline |tsoij-ts-o?o| & /tsoztsó?o/ & [tsoc'tsơ?o] & 'nuestros.EXCL dientes' \\
\hline$\left|\mathbf{i}_{1}-\mathrm{su}-\mathrm{x} \mathbf{i}\right|$ & /Esúx/ & {$\left[\varepsilon^{\prime} \operatorname{sux}\right]$} & 'su cara, su rostro' \\
\hline
\end{tabular}

Además, el sufijo |-tso?o|, que deriva los predicados afirmativos de formato y tamaño, parece afectar la última /i/ de la raíz a la que se agrega, como se muestra en el ejemplo 19.

(19) Descenso de $|\mathrm{i}|$ en raíces
a. /taßáctso?o/
[ta' $\beta_{T}$ actso?]
'es grande'
cf. [taß̦a' Pָic] 'grandeza'
b. /arußźctso?o/ [aru' $\beta_{\tau}^{\mathrm{w}} \boldsymbol{\varepsilon}$ :tso?]
'es grande (superficie)'
c. /aßáctso?o/
'es largo'
d. /pakoćtso?o/
[a'ßactso?]
'es ancho'
[aru $\beta_{T}^{w} a ' ?$ '
[aßa' ?̦ic] 'largura'
[t $\mathrm{fipa}$ 'koaijopi] 'no es ancho'

Sin embargo, el proceso no es totalmente automático, ya que las secuencias /is/, /its/ no

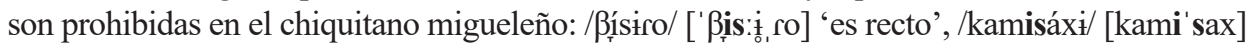
'camisa', /t jitsiropíti/ [t $\mathrm{t}$ itsiro' pitit] 'no se va'. ${ }^{17}$

\section{Elisión morfofonológica}

En algunos ambientes las vocales del chiquitano migueleño se pueden ensordecer o elidir. Esto ocurre tanto en el nivel posléxico (en este caso, la vocal puede realizarse como sonora bajo condiciones prosódicas específicas, así como, opcionalmente, en el habla cuidadosa o continua) como en el nivel léxico. En este apartado, discutiré solamente el proceso que opera en el nivel léxico: las vocales elididas no aparecen ni siquiera en el habla lenta y cuidadosa. Su presencia en la representación subyacente puede ser recuperada con base en la existencia de alomorfos con y sin la vocal (ejemplo 20), o bien en la ocurrencia de una de las consonantes $/ \underline{t}^{\mathrm{j}} \underline{\mathrm{c}} \mathrm{c} /$ en el comienzo de una palabra $\mathrm{o}$ tras una consonante (ejemplo 21). Repito que $/ \underline{\mathrm{t}} \underline{\mathrm{j}} \mathrm{c} / \mathrm{se}$ consideran derivadas de $|\mathrm{t} \mathrm{p} / \mathrm{k} \mathrm{x}|$ subyacentes mediante el proceso de la palatalización progresiva (véase 4), que puede ser desencadenado únicamente por una $\mid \mathrm{i}$ î| subyacente. Las vocales elididas son siempre altas y orales.

${ }^{17}$ Como ya se mencionó en el apartado 6 , el prefijo negativo/condicional tiene la forma / $\mathrm{t}$ i- $/$ solamente en el subdialecto de San Juan de Lomerío; en San Miguel y en otras comunidades este mismo prefijo tiene la forma $/ \mathrm{t} \mathrm{j}$ i-/. Ello podría explicar por qué su vocal /i/ no sufre el descenso a / $/ \varepsilon /$ delante de $/ \mathrm{s}$ ts/. 
(20) Elisión morfofonológica de vocales que aparecen en algunos alomorfos

\begin{tabular}{|c|c|c|c|c|}
\hline a. $|\mathrm{a}-(\beta) \mathrm{a}-\mathrm{riki}|$ & 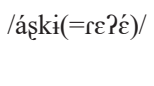 & {$\left[\right.$ aski( $(\ulcorner\varepsilon, \varepsilon)]^{18}$} & $\begin{array}{l}\text { 'hasta luego.ๆ' } \\
\text { (lit. 'acóstate pues. }{ }^{\prime} \text { ') }\end{array}$ & (cf. / ßaríkíro/ 'se acostó') \\
\hline b. $\mid i_{1}$-otopiriji-xi $\mid$ & /otoprijúxì/ & [otopri'jix] & 'su columna' & (cf. /otopíciji/ 'tu columna') \\
\hline c. $\mid i_{1}$-otuxiki-xi $\mid$ & /otuxkíci/ & [otux'kic] & 'su saliva' & (cf. /otúxiki/ 'tu saliva') \\
\hline d. |ixou-xi $\mid$ & /6oúxí/ & [6o'ux] & "víbora.甲' & (cf. /oicoúxí/ 'víbora. ô’) \\
\hline e. |ixine-xi| & /сinésí/ & [6ĩ' j̃es] & 'anguila. ${ }^{\prime}$ ' & (cf. /oicinési'/ 'anguila. ${ }^{\lambda}$ ') \\
\hline f. $\mid$ it $\int o \beta_{T} o r \varepsilon-x \dot{i} \mid$ & /tfoßorésí/ & {$\left[\mathrm{t} f \mathrm{o} \mathrm{\beta}_{\mathrm{T}}^{\prime} r \varepsilon \mathrm{s}\right]$} & 'diablo. + ' & 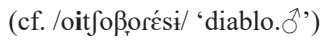 \\
\hline g. |o-kitapaki-xi| & /oktapakíci/ & [oktapa'kic] & 'anta. ${ }^{\lambda} '$ & (cf. /kitapakíci/ 'anta. \\
\hline h. |o-kituki-xí| & /oktukíci/ & [oktu'kic] & 'ajo (árbol). ${ }^{\lambda} ’$ & 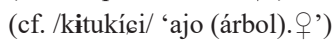 \\
\hline i. $\mid \varnothing$-ina ${ }^{2} a-t i \mid$ & /náati/ & ['na:tij] & ‘dijo’' & (cf. /omináati/ 'dijeron') \\
\hline j. $|o-s u t o n \varepsilon-x \dot{i}|$ & /ostonési/ & [osto'nes] & 'estrella. ${ }^{\lambda}$ ' & (cf. /sutonésì/ 'estrella. ${ }^{\prime}$ ') $)^{19}$ \\
\hline k. |utsau-xi| & /tsaúxí/ & [tsa'ux] & 'ayoreo.ㅇ' & 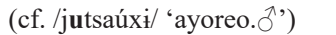 \\
\hline
\end{tabular}

(21) Palatalización causada por una |i| elidida
a. $\left|i_{1}-\max i \frac{p}{k} a ? i-m a ? a\right|$

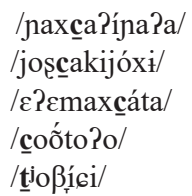
[naxca' P̦ina?]
'su gajo'
b. |jori $\frac{p}{k}$ akijo-xi $\mid$
[josçaki'jox]
'paquió'
c. $\mid \mathrm{a}-(\varepsilon ? \varepsilon) \max \mathbf{i}_{k}^{\frac{p}{k}}$ ata $\mid$
[ęmax' cata]
d. $\mid i_{k}^{\frac{p}{k}}$ Oõto?o $\mid$
e. |itoßị-xí|
['cótnto?]
'tu flojera'
f. |ituru-xí|

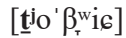
'luego'
[t. $\underline{\mathrm{j}}^{\mathrm{u}} \mathrm{u}^{\prime}$ rux]
'noche'
g. |ixina-nع|
/țurúxi/
[61̃ja'ne]
'puerta'
/ cinané/
[6orica'kic]
'frío'
h. |ixori ${ }_{k}^{p}$ aki-xí|
/coricakíci/
'chulupi, cucaracha'

Hay dos ambientes que favorecen la elisión de las vocales altas átonas: (i) $\mathrm{VC}_{1-} \mathrm{C}_{2} \mathrm{~V}$ (en que $\mathrm{C}_{1 / 2}$ es una consonante sorda o $|\mathrm{r}|$, sobre todo $\mathrm{si}_{1}$ es una fricativa) y (ii) \#_CV. No se trata de una regla absoluta sino de una tendencia, con morfemas distintos presentando comportamientos distintos. Por ejemplo, el prefijo $\left|i_{1}\right| 3$ SG se elide siempre y cuando el tema nominal/adposicional al que se agrega no es monomoraico (véase el apartado 5). Los prefijos $\left|i_{2}-\right|$ M. $\overbrace{}^{\hat{O}}$ y $\left|i_{2}-\right|$ INV se eliden siempre (palatalizando la consonante siguiente), como

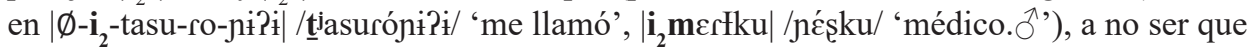
les preceda algún otro prefijo (|a-i-i_tasu-ka-ni?i| /aitiasukáni?i/ 'me llamaste'). Las vocales iniciales de raíces nominales pueden elidirse en la sílaba, como mínimo, antepretónica (|ixou-xí|/6oúxí/ 'víbora. + ', pero |ixi-xi| /icíci/ 'jichi, gusano, arcoíris. $\bigcirc$ '), aunque su elisión no es predecible (|inaká-xi|/inakáxí/ 'corechi. + ').

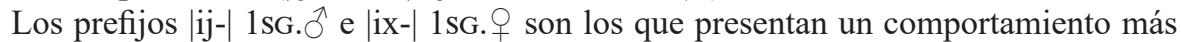
complejo. Como se muestra en el ejemplo 22, su vocal se elide regularmente si al prefijo

${ }^{18}$ La consonante $|\mathrm{f}|$, al silabificarse como una coda tras la elisión vocálica, suele transformarse en / $/$ (ver 21b). Lo único que impide el análisis de [s] como un alófono posicional de /f/ es la existencia de contadísimos préstamos del castellano en que $[\mathrm{c}] \mathrm{y}[\mathrm{s}$ ] excepcionalmente aparecen en coda y ataque, respectivamente:

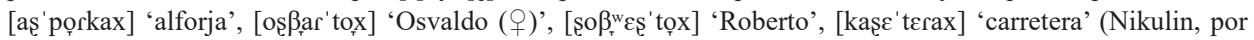
aparecer).

${ }^{19} \mathrm{La}$ forma del generolecto femenino /sutojési/ se ha atestiguado solamente en el subdialecto de San Miguel. En San Juan, el uso de la forma /ostonési/ se ha extendido del generolecto masculino para el femenino. 
le sigue uno de los prefijos |-( $\beta$ )a-| ANTP (22a-d) o |- $\mathrm{i}_{2}-\mid$ INV (22e-f). Si el prefijo se agrega directamente a un tema, la elisión dependerá (en parte) del segmento inicial del tema. La vocal se mantiene delante de $\left|\mathrm{p} \beta_{\mathrm{r}} \mathrm{m} \mathrm{s} \mathrm{t} \int\right|(22 \mathrm{~g}-1)$. Si el tema empieza por $|\mathrm{t}|(22 \mathrm{l}-\mathrm{o})$, $|\mathrm{k}|(22 \mathrm{p}-\mathrm{r})$ o recibe la consonante temática $|\mathrm{ts}|(22 \mathrm{~s}-\mathrm{v})$, la elisión o no de la vocal de los prefijos de $1 \mathrm{sg}$ está sujeta a variación léxica. Si el tema empieza por una vocal, |i| se mantiene en la posición pretónica $(22 \mathrm{w}-\mathrm{x})$ y se elide en la antepretónica $(22 \mathrm{y}-\mathrm{z})$.

(22) Elisión de |i| en los prefijos de 1sG

\begin{tabular}{|c|c|c|}
\hline a. $|i x-(\beta) a-t u ß a x i-k a|$ & /catuß̦axíça/ & 'molí. $\varphi^{\prime}$ \\
\hline b. |ij-( $\beta)$ a-tari-ma-ka| & /natanimáka/ & 'quebré. సै" \\
\hline c. $\mid$ ix $-(\beta) a-a^{2} a_{[+ \text {nas }]}-k a \mid$ & /cáka (kítu)/ & 'sembré. ㅇ' \\
\hline d. $\left|i j-(\beta) a-a^{2} a_{[+ \text {nas }]}^{[- \text {nas }}-k a\right|$ & /náka (kítu)/ & 'sembré. ${ }^{\lambda}$ ' \\
\hline e. $\left|i_{i}^{\frac{x}{i}-i_{2}}-\mathrm{ta} \beta a \mathrm{ai}-\mathrm{ta}\right|$ & /țaßáitịa/ & 'lo maté' \\
\hline f. $\quad\left|\frac{x}{i}-i_{2}-a^{2} a-t a\right|$ & /náta/ & 'lo puse' \\
\hline g. $\mid \mathbf{i}^{\frac{x}{i}}$-pakã?ã $\mid$ & /içakã́?ã/ & 'mi hígado' \\
\hline h. $\left|i^{\frac{x}{T}}-\beta a i t s i\right|$ & /ijáitsi/ & 'mi hamaca' \\
\hline i. $\left|i_{i}^{\frac{x}{i}}-\mathrm{masu}\right|$ & /inásu/ & 'mi oreja' \\
\hline j. $\quad \mid i \frac{x}{i}$-suto $\mid$ & /esúto/ & ‘mi ojo' \\
\hline 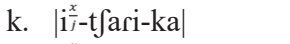 & /itfaríca/ & 'me peé' \\
\hline 1. $\mid \mathrm{i}_{\bar{t}}^{\mathrm{x}}$-toko-ka $\mid$ & /itfokóka/ & 'estoy bailando' \\
\hline m. $\mid$ ix-tiauxi $\mid$ & /itfiuuáuxi/ & 'mi hermano. ' \\
\hline n. $\mid \mathrm{i}^{\frac{x}{7}}$-turapa $\mid$ & /t furápa/ & 'mi amigo' \\
\hline o. $\mid \mathbf{i}^{\frac{x}{i}}$-tosi-ka $\mid$ & /t fosíka/ & 'tengo sed' \\
\hline 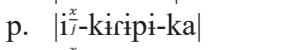 & /etsiripíka/ & 'tengo hambre' \\
\hline q. $\mid \mathrm{i}_{\overline{1}}^{\frac{x}{i}}$-kamisa $\mid$ & /etsamísa/ & 'mi camisa' \\
\hline r. $\quad\left|\mathrm{i}^{\frac{x}{y}}-\mathrm{ku} \beta_{\mathrm{T}} \mathrm{amoni}\right|$ & /tsußámoni/ & 'mi yerno' \\
\hline S. $\mid \mathrm{i}_{i}^{x}$-ts-o?o $\mid$ & /etsó?o/ & 'mi diente' \\
\hline t. $\mid i \frac{x}{i}$-ts-aruki $\mid$ & /tsarúki/ & 'mi hermano, mi hermana. ${ }^{\prime}$ ' \\
\hline u. $\left|\mathbf{i}_{i}^{\frac{x}{i}-\mathrm{ts}-\mathrm{o} \beta \mathrm{i} \mid}\right|$ & /tsóßì / & 'mediante mío' \\
\hline v. $\mid i_{i}^{\frac{x}{i}}$-ts-apa?u| & /tsápa?u/ & 'mi axila' \\
\hline w. $\mid$ ix-otu $_{[+ \text {nas }} \mid$ & /icótu/ & 'mi lengua. + ' \\
\hline x. $|i j-\tilde{\varepsilon} ? \tilde{\varepsilon}|^{[+ \text {nas }]^{\prime}}$ & 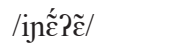 & "mi mano. ${ }^{\lambda}$ " \\
\hline y. |ix-ikiki $\mid$ & /6ikíki/ & 'mi uña. \\
\hline z. |ij-otopiriji & /jotopíriji/ & 'mi columna. ${ }^{\lambda}$ ' \\
\hline
\end{tabular}

El reconocimiento de la elisión vocálica como un proceso activo en chiquitano migueleño permite sustentar la hipótesis según la que absolutamente todos los encuentros consonánticos intramorfémicos provendrían de la elisión. Es decir, es posible proponer un análisis según el cual todas las sílabas en la representación subyacente serían abiertas (con la excepción de los prefijos de persona que terminan en consonante y pueden ser seguidos por raíces que empiezan por una consonante; en estos casos la consonante final del prefijo se elide). Sugiero que los ítems en el ejemplo 23 contienen una vocal alta no especificada (a pesar de que éstos no presentan alternancias sincrónicas entre vocales altas y el cero en la superficie), que represento como $|\mathrm{t}|$. 
(23) Elisión de vocales altas recuperable a partir de la fonotáctica

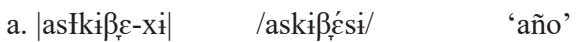
b. $\mid \operatorname{axft} \int a i-t s o$ ?o $\mid$ /axtfáctso?o/ 'tiene tos'

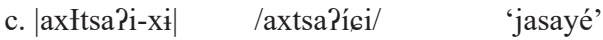
d. $\mid i_{1}$-axtt $\int u k i-x i \mid \quad$ /axtfukíci/ 'su suciedad (de un objeto)'

Esta solución analítica podría extenderse a los préstamos del castellano, como aquellos en $(23 \mathrm{e}-\mathrm{o})$.
e. |maixfticu|
/maíctiru/
'maestro. $\bigcirc$, maestra'
f. |arfporfka-xi|
/aspórkaxi/
'alforja'
g. $\mid$ arfkat $\varepsilon \mid$
/askáte/
'alcalde. + , alcaldesa'
h. |mesfku|
/mésku/
'médico. $\bigcirc$, médica'
i. |axfta ka?a|
/áxta ka?a/
'hasta que'
j. |kuxftan $\varepsilon \mid$
/kuxtáne/
'justán, enagua'
k. |kuexftaxi
/kućxtaxi//
'es difícil, cuesta'
1. |kaxftfrijaanu-xí|
/kaxtrijáanuxi//
'castellano'
m. $\left|\operatorname{apfro} \beta_{\tau} t \int a \beta_{\tau} 0\right|$
/aproßst $\beta_{\tau} \beta_{\tau} \mathrm{o} /$
'aprovechar'
n. $\left|\tilde{\varepsilon} \operatorname{tt} \varepsilon \beta_{\tau} \varepsilon r a \beta_{\tau} o\right|$

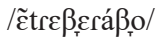
'entreverar, mezclar'

A pesar de que en el caso de los préstamos tal representación posiblemente no corresponde a la realidad diacrónica, el análisis propuesto aquí tiene la ventaja de eliminar la necesidad de postular encuentros consonánticos intramorfémicos en la representación subyacente.l

\section{Conclusión}

En este trabajo se discutieron los principales procesos morfofonológicos del chiquitano migueleño. Algunos de ellos coinciden con procesos atestiguados en las demás variedades del chiquitano (la nasalización, la africación, algunos tipos de la armonía vocálica, la elevación de $|\mathrm{o}| \mathrm{a} / \mathrm{u} /$ ), mientras que otros difieren significativamente de sus análogos en otros dialectos (la palatalización) o se describen en algún detalle por primera vez (el descenso de $|\mathrm{i}| \mathrm{a} / \varepsilon /$, la elisión morfofonológica). De esta manera, el presente trabajo, además de contribuir para la documentación de una variedad lingüística severamente amenazada e infradocumentada, puede presentar algún interés para las y los especialistas en la macrolengua chiquitana en general. En particular, una comprensión profundizada de la morfofonología del chiquitano migueleño contribuye para el entendimiento de la evolución histórica de los dialectos del chiquitano, por ser el análisis morfofonológico la primera etapa necesaria en la aplicación del método de la reconstrucción interna.

Estudios futuros deberán proponer un análisis unificado para la morfofonología de los prefijos de persona/número, así como para las instancias de “/ $\beta$ / móvil”, que desaparece en algunas fronteras morfológicas (véanse las notas 5 y 16). 


\section{NIKULIN - ELEMENTOS DE LA MORFOFONOLOGÍA...}

\section{Abreviaturas}

$q / \hat{\sigma}=$ habla femenina/masculina; $1 / 2 / 3=$ primera/segunda/tercera persona; ANTP $=$ antipasivo; $\mathrm{DIM}=$ diminutivo; $\mathrm{EXCL}=$ exclusivo; $\mathrm{F}=$ finito; $\mathrm{INCL}=$ inclusivo; $\mathrm{INV}=$ inverso; $\mathrm{M}=$ masculino; $\mathrm{NEG}=$ negativo; $\mathrm{NHA}=$ no humano, animado; $\mathrm{P}=$ argumento acusativo; $\mathrm{PL}$ $=$ plural; $\mathrm{PROH}=$ prohibitivo; $\mathrm{SG}=$ singular; $\mathrm{STAT}=$ estativo $\mathrm{C}=$ consonante $\mathrm{V}=$ vocal.

\section{Referencias}

Adam, Lucien; Henry, Victor (1880). Arte y vocabulario de la lengua chiquita. Con algunos textos traducidos y explicados compuestos sobre manuscritos inéditos del XVIII siglo. Paris: Maisonneuve y Cia.

Adelaar, Willem F. H. (2008). Relações externas do Macro-Jê. O caso do Chiquitano. In Stella V. T. de A. P. L. Telles; Aldir Santos de Paula (orgs.), Topicalizando Macro-Jê, pp. 9-28. Recife: Nectar.

Dietrich, Wolf (1986). El idioma chiriguano. Gramática, textos, vocabulario. Madrid: Instituto de Cooperación Iberoamericana.

Girard, Raphael (2014, mayo). Agreement without similarity: the case of Chiquitano nasal (consonant) harmony. Agreement by Correspondence-ABC, University of California-Berkeley, Berkeley.

González, Hebe Alicia (2014). Procesos fonológicos como rasgos areales: el caso de la palatalización en las lenguas chaqueñas. LIAMES. Linguas Indígenas Americanas 14: 11-39.

https://doi.org/10.20396/liames.v0i14.1519

Harris, John (1987). Non-structure-preserving rules in lexical phonology. Lingua 72(4): 255-92. https://doi.org/10.1016/0024-3841(87)90048-9

Kiparsky, Paul (1982). Lexical Morphology and Phonology. In Linguistic Society of Korea (ed.), Linguistics in the Morning Calm, pp. 3-91. Seul: Hanshin Publishing Co.

Krüsi, Martin; Krüsi, Dorothee (1978). Phonology of Chiquitano. In Work papers of the Summer Institute of Linguistics. Riberalta, Bolivia. 1972-1976, pp. 53-93. Riberalta: Summer Institute of Linguistics.

Nikulin, Andrey (2019a). First person singular markers in Migueleño Chiquitano. In D. K. E. Reisinger; Roger Yu-Hsiang Lo (eds.), Proceedings of the Workshop on the Structure and Constituency of Languages of the Americas 23, pp. 62-76. Vancouver: UBCWPL.

Nikulin, Andrey (2019b). Indexical gender and grammatical gender in Chiquitano. Typology of Morphosyntactic Parameters 2(1): 86-99.

Nikulin, Andrey (2019c). Contacto de lenguas en la Chiquitanía. Revista Brasileira de Línguas Indígenas 2(2): 5-30.

Nikulin, Andrey (por aparecer). Fonología segmental del chiquitano migueleño. Boletim do Museu Paraense Emílio Goeldi. Ciências Humanas.

Nikulin, Andrey (entregado para publicación). La fonología del acento en el chiquitano migueleño. 
Parapaino Castro, Pablino (2008). Isiukiché nikorokó Bésiro. Guía de escritura del idioma Bésiro. Santa Cruz de la Sierra: Unión de Artesanos de la Tierra-UNIARTE.

Ramirez, Henri; Vegini, Valdir; França, Maria Cristina Victorino de (2017). O warázu do Guaporé (tupi-guarani): primeira descrição linguística. LIAMES. Linguas Indigenas Americanas 17(2): 411-506. https://doi.org/10.20396/liames.v17i0.8647468

Sans, Pierric (2010). Éléments de sociolinguistique et de phonologie du bésiro (chiquitano). Langue en danger des basses terres de Bolivie (Tesis de maestría en Lingüística). Lyon: Université Lumière Lyon 2.

Sans, Pierric (2011). Is nasality an autosegmental feature in Bésiro (a.k.a. Chiquitano)? In Ana Suelly Arruda Câmara Cabral; Aryon Dall'Igna Rodrigues; Jorge Domingues Lopes; Maria Risolêta Silva Julião (orgs.), Línguas e culturas Tupí, v. 3, Linguas e culturas Macro-Jê, v. 2, pp. 237-48. Campinas: Curt Nimuendajú; Brasília: LALI/UnB.

Silva, Mário André Coelho da (2020a). Tikmũũg ỹ̃y ax tinã xohi xi xahĩnãg. Sons e pedaços da língua Maxakalí: descrição da fonologia e morfologia de uma língua Macro-Jê (Tesis doctoral en Lingüística). Belo Horizonte: Universidade Federal de Minas Gerais.

Silva, Mário André Coelho da (2020b). Um novo olhar sobre as formas longas e curtas em nomes no Maxakalí. In Marcelo Silveira; Maria José Guerra; Ludoviko dos Santos (orgs.), Macro-Jê: língua, cultura e reflexões, p. 147-62. Londrina: Editora da Universidade Estadual de Londrina.

Recebido: $13 / 8 / 2020$

Versão revista: 13/10/2020

Aceito: 16/10/2020

Publicado: 26/10/2020 